\title{
Stepwise illustration of teeth-fixation semi-constrained cervical disc arthroplasty
}

\author{
Chih-Chang Chang, MD, ${ }^{1,2}$ Jau-Ching Wu, MD, PhD, ${ }^{1,2}$ Peng-Yuan Chang, MD, ${ }^{4}$ Mei-Yin Yeh, MD, ${ }^{1,2}$ \\ Yi-Hsuan Kuo, MD, , ${ }^{1,2}$ Li-Yu Fay, MD, 1,2 Tsung-Hsi Tu, MD, ${ }^{1-3}$ Wen-Cheng Huang, MD, PhD, 1,2 \\ and Henrich Cheng, MD, PhD ${ }^{1,2}$
}

\begin{abstract}
1Department of Neurosurgery, Neurological Institute, Taipei Veterans General Hospital; ${ }^{2}$ School of Medicine, National Yang-Ming University; ${ }^{3}$ Taiwan International Graduate Program in Molecular Medicine, National Yang-Ming University and Academia Sinica, Taipei; and ${ }^{4}$ Department of Neurosurgery, Taoyuan General Hospital, Ministry of Health and Welfare, Taoyuan City, Taiwan
\end{abstract}

There are many kinds of artificial discs available for cervical disc arthroplasty (CDA), with various designs of fixation and articulation mechanisms. Each of these designs has different features and theoretically fits most optimally in selected types of patients. However, there has been insufficient literature to guide individualized selection among these CDA devices. Since CDA aims to restore the joint function rather than arthrodesis, tailor-made size, shape, and mechanical properties should be taken into account for each candidate's target disc. Despite several large-scale prospective randomized control trials that have demonstrated the effectiveness and durability of CDA for up to 8 years, none of them involved more than one kind of artificial disc. In this video the authors present detailed steps and technical aspects of the newly introduced ProDisc-C Vivo (DePuy Synthes Spine), which has the same ball-and-socket design for controlled, predictable motion as the ProDisc-C. The newly derived teeth fixation provides high primary stability and multilevel capability by avoidance of previous keel-related limitations and complications (e.g., split vertebral fracture). Please note that the ProDisc-C Vivo is currently not available on the US market.

The authors present the case of a 53 -year-old woman who had symptoms of both radiculopathy and myelopathy caused by a large, calcified disc herniation at C4-5. There was no improvement after 4 months of medical treatment and rehabilitation. A single-level CDA was successfully performed with the ProDisc-C Vivo, and her symptoms were completely ameliorated afterward. The follow-up images demonstrated preservation of motion at the indexed level.

The video can be found here: https://youtu.be/4DSES1xgvQU.

KEYWORDS cervical disc; arthroplasty; semi-constrained; disc herniation; spondylosis; video 